\title{
Safety and Tolerability of Edivoxetine for Long-Term Treatment of Major Depressive Disorder in Adult Patients
}

\author{
Tina M. Oakes ${ }^{1 *}$, James M. Martinez ${ }^{1}$, Mary Anne Dellva ${ }^{1}$, Celine Goldberger ${ }^{1}$, \\ Beth A. Pangallo', Mark E. Bangs ${ }^{1}$, Jonna Ahl', William B. White ${ }^{2}$ \\ ${ }^{1}$ Eli Lilly and Company, Indianapolis, USA \\ ${ }^{2}$ University of Connecticut School of Medicine, Farmington, USA \\ Email: oakes tina marie myers@lilly.com
}

Received 13 March 2014; revised 7 April 2014; accepted 14 April 2014

Copyright (C) 2014 by authors and Scientific Research Publishing Inc.

This work is licensed under the Creative Commons Attribution International License (CC BY).

http://creativecommons.org/licenses/by/4.0/

(c) (i) Open Access

\section{Abstract}

This 12-month open-label, but dose-blinded extension phase, evaluated the safety and tolerability of flexibly-dosed edivoxetine $(6,9,12$ or $18 \mathrm{mg}$ once daily) in patients $(\mathrm{N}=397)$ with major depressive disorder, who completed the 10-week randomized, double-blind, placebo-controlled acute phase of the study. All patients were treated with edivoxetine during the extension phase. The mean age of the patients was 45 years, and most were white females. Safety evaluations included assessment of treatment-emergent adverse events (TEAEs), laboratory and vital sign measures, and suicidality. Within-group t-tests based on a 2 -sided significance level of 0.05 and $95 \%$ confidence levels were used to assess whether changes from baseline were statistically significant from zero. The overall completion rate was 54\%. Adverse event was the most common (14.4\%) reason for discontinuation, which included blood pressure increased $(1.3 \%)$, heart rate increased $(1.3 \%)$, anxiety (1.0\%), and tachycardia (1.0\%). At least 1 TEAE was reported by $72.3 \%$ of patients, of which headache $(10.8 \%)$ and hyperhidrosis $(10.1 \%)$ were the most common; $2.8 \%$ of patients had $\geq 1$ serious adverse events, and there were no completed suicides. No clinically relevant changes were observed in most laboratory measures. Potentially clinically significant changes in ALT values occurred in $1.8 \%$ of patients, and either normalized or had decreased by the last assessment. Mean increases in blood pressure and pulse were consistent with those observed in the acute phase and appeared to reach a plateau within 3 to 5 months of treatment. In conclusion, safety and tolerability findings during this long-term extension phase evaluation of edivoxetine were consistent with its norepinephrine reuptake inhibition profile.

*Corresponding author.

How to cite this paper: Oakes, T.M., et al. (2014) Safety and Tolerability of Edivoxetine for Long-Term Treatment of Major Depressive Disorder in Adult Patients. Open Journal of Psychiatry, 4, 131-140. 


\section{Keywords}

\section{Edivoxetine, Norepinephrine, Major Depressive Disorder, Safety, Tolerability}

\section{Introduction}

Major depressive disorder is a chronic and debilitating disorder that usually requires long-term treatment [1]. Patients with MDD often experience relapses and recurrences, and it is estimated that only a third of patients receiving antidepressant therapy achieve full remission with monotherapy [2]. After acute treatment (up to 12 weeks) as many as one-third to one-half of MDD patients who initially responded, will relapse if treatment is not continued for at least 3 - 9 months beyond acute symptom resolution [1]. Successful long-term treatment is dependent, in part, on patient and physician education regarding the nature of depression, as well as the long-term safety and tolerability of antidepressant treatment [3].

All currently available antidepressants affect neurotransmission of at least one of these three neurotransmitters: serotonin, norepinephrine, and dopamine. Globally, these antidepressants include monoamine oxidase inhibitors, tricyclic antidepressants, selective serotonin reuptake inhibitors (SSRI), serotonin-norepinephrine reuptake inhibitors (SNRI), selective norepinephrine reuptake inhibitors (NRI), and other agents [3] [4].

Edivoxetine is a highly selective and potent NRI that is being studied for the treatment of MDD. This was a 62-week study of flexibly-dosed edivoxetine in patients with MDD that was comprised of a 10-week doubleblind placebo-controlled acute phase followed by a 52-week open-label extension phase. The acute phase has been published [5]; herein we present the safety and tolerability results of the extension phase.

\section{Materials and Methods}

\subsection{Overview}

This was a 62-week Phase II/III multicenter study with a 10-week randomized, double-blind, placebo-controlled, flexible-dose, parallel-arm acute treatment phase comparing edivoxetine 6, 9, 12, and 18 mg once daily (QD) with placebo, followed by a 1-year open-label but dose-blinded, flexible-dose extension phase, and a 2-week taper off drug phase. The study was conducted at 43 sites across five countries: the United States, Finland, Poland, Russia, and Argentina. Enrollment for the acute phase began in November 2008, and the 1-year extension phase was completed in March 2011. The institutional review boards approved the protocol and all patients provided written informed consent. This study was conducted in accordance with ethical principles that have their origin in the Declaration of Helsinki, and are consistent with Good Clinical Practices and applicable laws and regulations.

\subsection{Patient Selection}

The study included female and male outpatients aged 18 to 65 years who met criteria for MDD without psychotic features, as defined by the Diagnostic and Statistical Manual of Mental Disorders, Fourth Edition Text Revision (DSM-IV-TR) (APA, 2000) and confirmed by the Mini-International Neuropsychiatric Interview (MINI) [6]. Patients were required to have a GRID 17-item Hamilton Rating Scale for Depression (GRIDHAMD $_{17}$ ) [7] total score $\geq 18$, and Clinical Global Impressions-Severity (CGI-S) [8] score $\geq 4$.

Key exclusion criteria included: any additional ongoing DSM-IV-TR Axis I condition other than major depression or any anxiety disorder (excluding specific phobias) preceding the onset of depression, which was considered the primary diagnosis within 1 year prior to enrollment in the acute phase of the study. Patients were also excluded if they were judged by the investigator to be at risk for harm to self or others. Patients were also to be excluded if they had an unstable medical condition or had any diagnosed medical condition, which could be exacerbated by noradrenergic agents including unstable hypertension or unstable heart disease, tachycardia or tachyarrhythmia, narrow angle glaucoma, or urinary hesitancy or retention.

\subsection{Study Design}

All patients who completed the acute phase of the study were eligible to enter the 1-year open-label dose- 
blinded extension phase (Figure 1). Patients randomized to edivoxetine during the acute phase entered the extension phase on their current blinded dose of edivoxetine. Patients randomized to placebo during the acute phase began dose-blinded treatment in the 1-year extension phase with edivoxetine $6 \mathrm{mg}$ QD and were titrated to 9 mg QD after 1 week. Even though investigators and patients were blinded to the dose of edivoxetine, investigators were given the option to request a dose increase at scheduled visits, or a dose decrease at any time based on treatment response and safety and tolerability. For all patients in the extension phase, the patient's dose was to be increased if the CGI-S score was $\geq 3$ unless the patient was unable to tolerate an increased dose. If the CGI-S score was $<3$ and the patient was tolerating the current dose, the investigator could increase the dose to optimize response. Patients unable to tolerate an increased dose could be returned to the previously tolerated dose. However, if unable to tolerate that dose, investigators used their clinical judgment on whether to continue that dose or discontinue the patient's participation in the study. In the 2-week taper phase, patients who completed the extension phase of the study or discontinued early from the study for any reason were tapered as follows: patients whose current dose was edivoxetine $18 \mathrm{mg}$ received $12 \mathrm{mg}$ QD for 1 week followed by $6 \mathrm{mg}$ QD for 1 week; patients whose current dose was edivoxetine 12, 9, or $6 \mathrm{mg}$ received $6 \mathrm{mg}$ QD for 2 weeks.

\subsection{Outcome Measures and Assessments}

Safety and tolerability assessments included spontaneously reported treatment-emergent adverse events (TEAEs), serious adverse events (SAEs), discontinuation rates, clinical laboratory tests (chemistry, hematology, and urinalysis), vital signs (blood pressure, pulse, weight), and electrocardiograms (ECGs). The occurrence, severity, and frequency of suicide-related events (behavior and/or ideation) were assessed using the Columbia Suicide Severity Rating Scale (C-SSRS) [9], as well as unsolicited adverse event reporting.

Measures to evaluate the effect of edivoxetine in improving depression and related symptoms included: the Montgomery-Åsberg Depression Rating Scale (MADRS) [10]; CGI-S; Fatigue Associated with Depression questionnaire (FAsD) [11]; and Sheehan Disability Scale (SDS) [12]. Response was defined as at least a 50\% reduction from baseline on the MADRS total score. Remission was defined as a MADRS total score $\leq 10$.

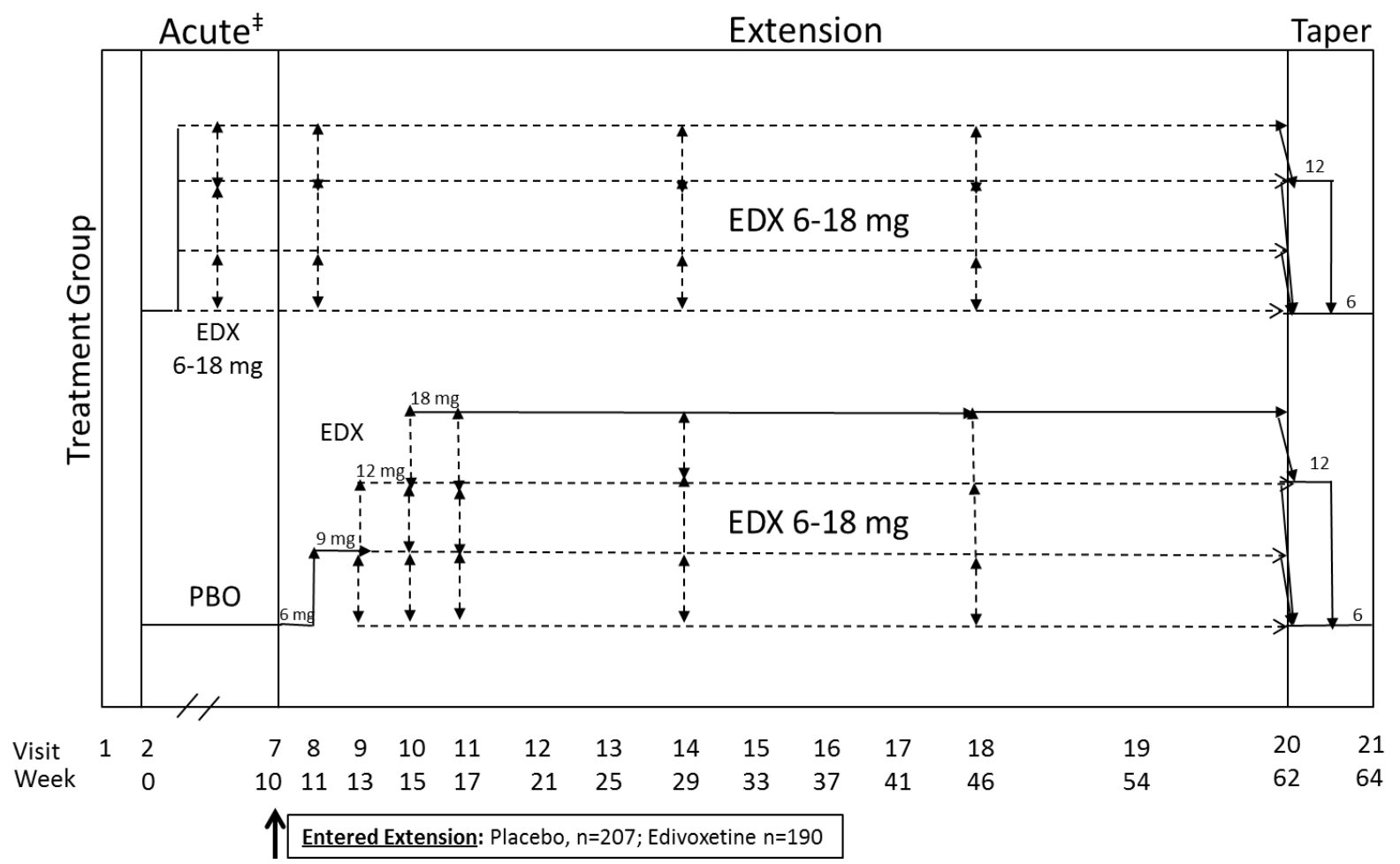

${ }^{\ddagger}$ Details of EDX titration during the acute phase have been described previously (Pangallo et al., 2011) and were similar for PBO patients switching to EDX during the extension phase.

Figure 1. Study Design. Abbreviations: EDX, edivoxetine; PBO, placebo. 


\subsection{Statistical Analysis}

All analyses were conducted on an intent-to-treat basis, and included only those patients who entered the 1-year extension phase. Baseline for analysis of safety measures, as well as efficacy and health outcomes, was the last visit in the acute treatment phase at Week 10. For changes in ECGs and laboratory tests, the last nonmissing value from the acute phase, including unscheduled visits, was utilized as the baseline. Because all patients received edivoxetine in the extension phase, results were subgrouped and summarized by the acute phase treatment assignments. Patients randomly assigned to placebo during the acute phase were referred to as the placebo/edivoxetine group (PBO/EDX), and patients randomly assigned to edivoxetine during the acute phase were referred to as the edivoxetine/edivoxetine (EDX/EDX) group. No statistical comparisons between these groups were conducted. Within-group t-tests based on a 2-sided significance level of 0.05 and $95 \%$ confidence intervals were used to assess whether changes from baseline were statistically significantly different from zero. The modal, mean, maximum, and last doses were summarized by treatment group for each study period.

Change from baseline for continuous safety, efficacy, and health outcomes measures were analyzed using a restricted maximum likelihood based mixed-effects model repeated measures analysis (MMRM) for each group. The model for this analysis included the fixed class effects of pooled investigative site and visit, and the continuous, fixed covariates of baseline score and baseline score-by-visit interaction. The percentages of patients meeting response and remission criteria were summarized at each observed visit and at the last-observation- carried-forward (LOCF) endpoint. The percentages of patients meeting criteria for categorical safety variables, including TEAEs, SAEs, adverse events reported as reason for requesting dose reduction and/or discontinuation, and categorical criteria for changes in vital signs, weight, ECGs, and laboratory tests, were summarized by group. The percentages for gender-specific adverse events were adjusted for the appropriate gender.

Categorical treatment-emergent changes in vital signs were summarized for patients who did not meet threshold criteria at baseline. Treatment-emergent orthostatic changes meeting criteria for orthostatic hypotension were defined as a systolic blood pressure decrease of $\geq 20 \mathrm{~mm} \mathrm{Hg}$ from supine to standing or a pulse increase of $\geq 20$ beats per minute (bpm) from supine to standing. Sustained elevations in systolic blood pressure were defined as 3 consecutive post-baseline values elevated $\geq 10 \mathrm{~mm} \mathrm{Hg}$ from baseline and meeting at least $140 \mathrm{~mm} \mathrm{Hg}$ threshold. Sustained elevations in diastolic blood pressure were defined as 3 consecutive post-baseline values that were $\geq 10 \mathrm{~mm} \mathrm{Hg}$ from baseline and met a threshold of at least $90 \mathrm{~mm} \mathrm{Hg}$. Potentially clinically significant (PCS) elevations in blood pressure and pulse were defined as systolic blood pressure $\geq 180 \mathrm{~mm} \mathrm{Hg}$ and $\geq 20 \mathrm{~mm}$ $\mathrm{Hg}$ increase from baseline; diastolic blood pressure $\geq 105 \mathrm{~mm} \mathrm{Hg}$ and $\geq 15 \mathrm{~mm} \mathrm{Hg}$ increase from baseline; or pulse rate $>120 \mathrm{bpm}$ and $\geq 15 \mathrm{bpm}$ increase from baseline. PCS changes in weight were defined as a decrease of at least $7 \%$ of body weight or an increase of at least $7 \%$ of body weight.

\section{Results}

\subsection{Patient Disposition}

Of the 408 patients who completed the acute treatment phase, a total of 397 patients, including 207 patients from the placebo treatment group and 190 from the edivoxetine treatment group, entered the 1-year extension phase (Figure 2). Of 397 patients, 54\% completed the extension phase. The most frequent reason for discontinuation was adverse event (14.4\%), followed by patient decision (12.6\%). Approximately $60 \%$ of the patients who entered the 1-year extension phase also entered the taper phase. The majority of those patients (97\%) completed the taper phase.

\subsection{Patient Demographics and Illness Severity}

Demographics for the patients who entered the 1-year extension phase are shown in Table 1. The majority of patients were white, female, and less than 55 years of age, with a mean overall age of 44.9 years. The severity of illness for patients at the beginning of the extension phase is shown in Table 2. Patients who received placebo in the acute phase had numerically higher severity scores on each measure than those who received edivoxetine.

\subsection{Dosing}

Across both groups compliance with treatment during the 1-year extension phase was $74.4 \%$. The mean length 


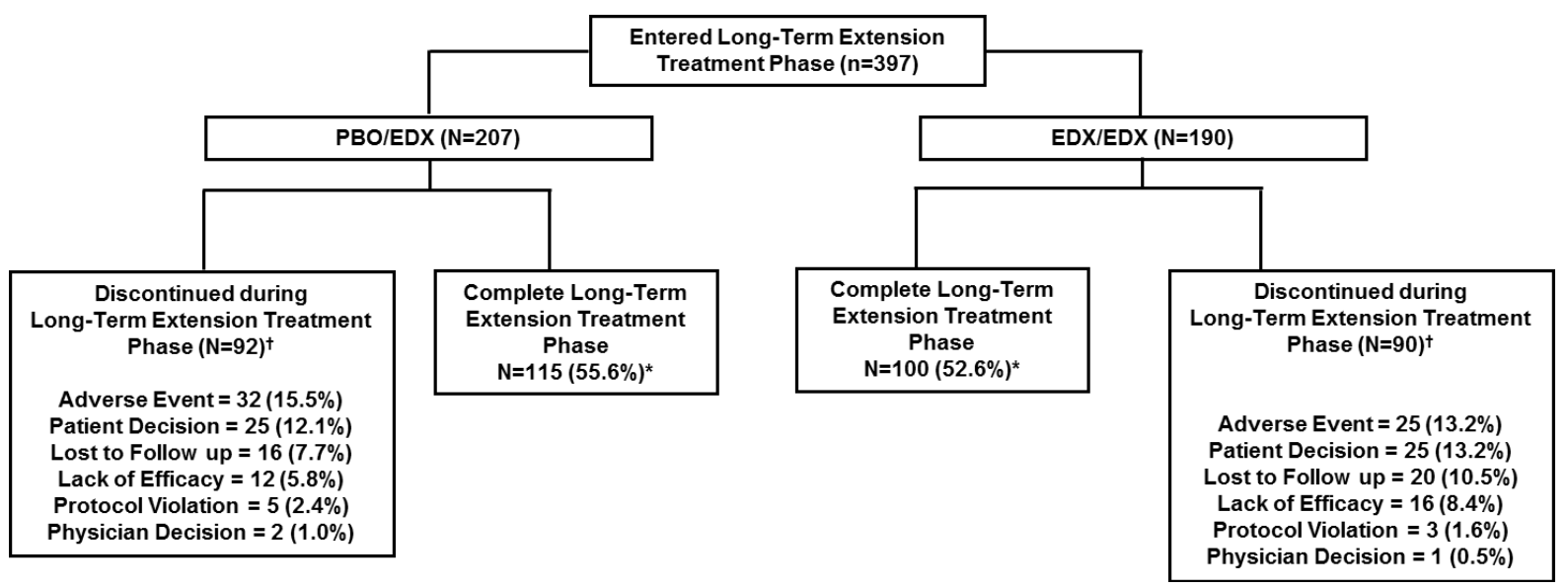

Figure 2. Patient disposition. Abbreviations: EDX, edivoxetine; PBO, placebo.

Table 1. Demographics of patients entering the extension phase.

\begin{tabular}{ccc}
\hline Variable & PBO/EDX (N = 207) & EDX/EDX (N = 190) \\
\hline Age, mean years (SD) & $45.8(11.53)$ & $44.0(11.39)$ \\
$<55$ years, n (\%) & $156(75.4)$ & $158(83.2)$ \\
$\geq 55$ years, n (\%) & $51(24.6)$ & $32(16.8)$ \\
Female, n (\%) & $120(58.0)$ & $120(63.2)$ \\
Race, n (\%) & $162(78.3)$ & $34(76.8)$ \\
White & $33(15.9)$ & $0(0.0)$ \\
Black & $3(1.4)$ & $2(1.1)$ \\
American Indian or Alaska Native & $2(1.0)$ & $7(3.7)$ \\
Asian & $5(2.4)$ & $0(0.0)$ \\
Multi-racial & $1(0.5)$ & $1(0.5)$ \\
Other & $1(0.5)$ & \\
\hline
\end{tabular}

Abbreviations: EDX/EDX, edivoxetine/edivoxetine; PBO/EDX, placebo/edivoxetine; SD, standard deviation.

Table 2. Severity of illness scores of patients entering the extension phase.

\begin{tabular}{ccccc}
\hline Measure & $\mathrm{N}$ & PBO/EDX Mean, SD & $\mathrm{N}$ & EDX/EDX Mean, SD \\
\hline MADRS Total & 202 & $19.9(8.9)$ & 185 & $15.9(8.9)$ \\
CGI-Severity & 207 & $3.4(1.1)$ & 188 & $3.0(1.1)$ \\
FAs-D PRO Average & 207 & $2.8(1.0)$ & 185 & $2.6(0.9)$ \\
FAs-D Experience & 207 & $3.0(1.0)$ & 187 & $2.7(1.0)$ \\
FAs-D Impact & 207 & $2.7(1.0)$ & 186 & $2.4(0.9)$ \\
SDS, Global & 186 & $13.7(7.8)$ & 179 & $10.9(7.5)$ \\
\hline
\end{tabular}

Baseline severity of illness for the extension phase was derived as the Week 10 value from the acute phase of the study. $\mathrm{N}$ refers to the number of patientswith non-missing data for the given variable at both baseline and at least one post-baseline visit. Abbreviations: CGI-S, Clinical Global Impression of Severity; EDX, edivoxetine; FAs-D PRO, Fatigue Associated with Depression Patient Reported Outcome; MADRS, Montgomery-Åsberg Depression Rating Scale; PBO, placebo; SD, standard deviation; SDS, Sheehan Disability Scale.

of exposure to edivoxetine in the PBO/EDX group was approximately 250 days, and for the EDX/EDX group it was approximately 259 days. The mean modal dose of edivoxetine across the 52 weeks of the extension phase treatment was $12.2 \mathrm{mg} /$ day (median $=9.0 \mathrm{mg}$ ) for the PBO/EDX group and $14.7 \mathrm{mg} /$ day (median $=18.0 \mathrm{mg}$ ) for the EDX/EDX group. Among study completers, the frequency distribution of patients at each final edivoxetine dose was 13.9\% (6 mg QD), 26.1\% (9 mg QD), 16.5\% (12 mg QD), and 43.5\% (18 mg QD) in the PBO/EDX group; and 7.5\% (6 mg QD), 16.0\% (9 mg QD), 18.9\% (12 mg QD), and 57.5\% (18 mg QD) in the EDX/EDX group. 


\subsection{Adverse Events}

TEAEs reported by at least $5 \%$ of patients are summarized in Table 3 . Overall, $72.3 \%$ of patients reported at least 1 TEAE. Headache (10.8\%) and hyperhidrosis (10.1\%) were most commonly reported with a numerically higher incidence in patients who switched from placebo to edivoxetine (13.0\% and $13.5 \%$, respectively) compared with patients who continued on edivoxetine (8.4\% and 6.3\%, respectively). Rates of discontinuation due to an adverse event were 15.5\% in the PBO/EDX group and 13.2\% in the EDX/EDX group. Across both groups, the most common adverse events leading to discontinuation were blood pressure increased (1.3\%), heart rate increased (1.3\%), anxiety (1.0\%), and tachycardia (1.0\%). Overall, $23.7 \%$ of patients reported an adverse event as a reason for requesting a reduction in dose. The most common events were erectile dysfunction (3.2\% of males), tachycardia (3.0\%), heart rate increased (2.3\%), and hyperhidrosis (2.3\%). During the taper phase, 15 out of 236 (6.4\%) patients experienced at least one taper-emergent adverse event. The most common events were constipation and irritability, and each event was reported by 2 patients $(0.8 \%)$.

The frequency of patients with at least $1 \mathrm{SAE}$ was $2.8 \%$, and there were no deaths in the extension phase. The SAEs reported for more than 1 patient were suicide attempt (2 patients in the PBO/EDX group) and non-cardiac chest pain (2 patients in the EDX/EDX group). SAEs reported by one patient each in the PBO/EDX group were gastroenteritis, intentional overdose, lumbar radiculopathy, schizoaffective disorder, and spinal osteoarthritis; and the EDX/EDX group reported drug abuse, hip fracture, musculoskeletal chest pain, myocardial infarction, tachycardia paroxysmal, and missed abortion. Three patients reported multiple SAEs as follows: In the PBO/ EDX group one patient had lumbar radiculopathy and spinal osteoarthritis; another patient reported an intentional overdose and suicide attempt. In the EDX/EDX group, one patient experienced a hip fracture and noncardiac chest pain, which were reported at different times.

In addition to the suicide attempt reported as an SAE, treatment-emergent suicidal ideation and behavior were reported on the C-SSRS. Eleven (5.3\%) patients in the PBO/EDX group and 7 (3.7\%) patients in the EDX/EDX group reported having treatment-emergent suicidal ideation. C-SSRS treatment-emergent suicidal behavior was reported by 1 (0.5\%) patient in the PBO/EDX group and 2 (1.1\%) patients in the EDX/EDX group.

\subsection{Laboratory Measures}

Statistically significant within-group differences were observed for LS mean change from extension phase baseline at various time points for some chemistry, hematology, and urinalysis laboratory tests. Most of the changes were small and not considered clinically relevant. No patient had a serious adverse event related to abnormal laboratory values.

Seven patients had elevated alanine transaminase (ALT) ( $\geq 3$ times the upper limit of normal, ULN) and 2 had elevated total bilirubin ( $\geq 2$ times the ULN). None of the patients met both ALT and total bilirubin elevation criteria (Hy's rule) at anytime. Of those patients who had ALT elevations, 2 were in the EDX/EDX group and 5 were in the PBO/EDX group. Both of the patients in the EDX/EDX group entered the extension phase with normal ALT levels. One of these patients had ALT levels that were $\geq 3$ times the ULN at Week 62, which re-

Table 3. Treatment-emergent adverse events occurring in at least $5 \%$ of patients in the extension phase.

\begin{tabular}{|c|c|c|c|}
\hline Event & PBO/EDX N = 207 & EDX/EDX N =190 & Total $\mathrm{N}=397$ \\
\hline & $\mathrm{n}(\%)$ & n (\%) & $\mathrm{n}(\%)$ \\
\hline Patients with at least 1 event & $158(76.3)$ & 129 (67.9) & $287(72.3)$ \\
\hline Headache & $27(13.0)$ & $16(8.4)$ & $43(10.8)$ \\
\hline Hyperhidrosis & $28(13.5)$ & $12(6.3)$ & $40(10.1)$ \\
\hline Nausea & $23(11.1)$ & $13(6.8)$ & $36(9.1)$ \\
\hline Erectile dysfunction & $9(10.3)$ & $1(1.4)$ & $10(6.4)$ \\
\hline Nasopharyngitis & $18(8.7)$ & $7(3.7)$ & $25(6.3)$ \\
\hline Tachycardia & $17(8.2)$ & $8(4.2)$ & $25(6.3)$ \\
\hline Insomnia & $14(6.8)$ & $9(4.7)$ & $23(5.8)$ \\
\hline Heart rate increased & $13(6.3)$ & $9(4.7)$ & $22(5.5)$ \\
\hline Upper respiratory tract infection & $10(4.8)$ & $10(5.3)$ & $20(5.0)$ \\
\hline Constipation & $12(5.8)$ & $6(3.2)$ & $18(4.5)$ \\
\hline
\end{tabular}


turned to normal levels during the 2-week taper-off drug period. The other patient in the EDX/EDX group had ALT levels that were $\geq 3$ times the ULN at Week 25 that returned to normal within 11 days, and continued to be normal at Week 62. In the opinion of the investigator, the elevation in ALT levels in that patient may have been associated with alcohol intake. In the PBO/EDX group, 2 patients discontinued early and had ALT levels that were $\geq 3$ times the ULN, which returned to normal after discontinuation of edivoxetine. Two other patients in the PBO/EDX group had elevated ALT levels that were possibly associated with alcohol use in the opinion of the investigator. Even though the ALT levels in these two patients had decreased, both were discontinued early; one due to physician decision, and the other due to experiencing an adverse event of increased blood pressure. The fifth patient in the PBO/EDX group had normal ALT levels at extension phase entry and the levels were $\geq 3$ times the ULN at Week 25, which the investigator thought was associated with excessive exercise. After this patient completed the study, ALT levels returned to within normal limits. Of the 2 patients with elevated total bilirubin, both were in the EDX/EDX group and had elevated levels prior to extension phase entry. One patient completed the trial; the other discontinued early as the elevated total bilirubin was attributed to Gilbert's syndrome.

\subsection{Vital Signs and Weight}

Changes from extension phase entry to Week 62 in vital signs and weight are summarized in Table 4. Patients in the PBO/EDX group had significant within-group mean increases in supine systolic and diastolic blood pressure and pulse, whereas patients in the EDX/EDX group did not. There were no significant within-group changes in weight in either group. The percentages of patients with PCS changes in vital signs and weight are summarized in Table 5. Only two patients met PCS criteria for both systolic and diastolic blood pressure changes. The percentages of patients with PCS changes in weight were numerically greater for weight loss than for weight gain in both PBO/EDX and EDX/EDX groups. Elevations in supine blood pressures that were sustained over 3 consecutive visits were experienced by $11.6 \%$ of PBO/EDX-treated patients for systolic blood pressure and 6.5\% for diastolic blood pressure. For patients in the EDX/EDX group, 4.7\% experienced sustained elevations in systolic blood pressure and $7.7 \%$ had sustained elevations in diastolic blood pressure. Treatmentemergent orthostatic changes in blood pressure and pulse, respectively, were experienced by $13.3 \%$ and $15.9 \%$ of patients in the PBO/EDX group and $10.2 \%$ and $17.2 \%$ of patients in the EDX/EDX group. No patient with orthostatic blood pressure or pulse changes experienced a fall. Two patients with orthostatic pulse increases discontinued due to tachycardia and heart rate increased, respectively, and four patients with orthostatic blood pressure changes discontinued due to elevated blood pressure.

At Week 62, ECG changes from extension phase entry in either group were not clinically significant as assessed by Fridericia's corrected QT interval (QTcF). None of the patients had an increase in QTcF of >60 msec from baseline or experienced a QTcF $\geq 500$ msec.

\subsection{Efficacy Outcomes}

Changes from extension phase entry in illness severity are summarized in Table 6. Statistically significant within-group mean improvements (reductions) in the MADRS total at Week 62 were observed for patients in both PBO/EDX and EDX/EDX groups. In addition, overall symptom severity was significantly reduced as assessed by changes in the CGI-S and measures of fatigue and functioning. Response rates at LOCF endpoint (from baseline which was Week 10 of the acute phase) were $47.0 \%$ and $43.6 \%$, respectively, for the PBO/EDX and EDX/EDX groups; remission rates were $53.0 \%$ and $60.5 \%$, respectively.

\section{Discussion}

This open-label extension phase evaluated the long-term safety and tolerability of flexibly-dosed edivoxetine in adult patients with MDD over 52 weeks. There were no new significant findings, as the outcomes were consistent with the results of the double-blind, placebo-controlled 10-week acute phase study [5]. That edivoxetine has long-term tolerability for use in treatment of MDD was supported by a high completion rate (54\%) and high overall compliance with study medication (74.4\%), as well as a $14.4 \%$ rate of discontinuation due to adverse events. Although approximately $72 \%$ of patients reported TEAEs, most events were considered mild or moderate in severity. In general, the TEAEs reported by the PBO/EDX group occurred with numerically greater fre- 
Table 4. LS mean changes from the extension phase baseline ${ }^{a}$ in vital signs and weight.

\begin{tabular}{ccccccc}
\hline & \multicolumn{3}{c}{ PBO/EDX } & \multicolumn{2}{c}{ EDX/EDX } \\
\hline Measure & $\begin{array}{c}\text { LS Mean Change, } \\
\text { (SE) }\end{array}$ & CI & $\begin{array}{c}\text { Within-group } \\
P \text { values }\end{array}$ & $\begin{array}{c}\text { LS Mean Change, } \\
\text { (SE) }\end{array}$ & CI & $\begin{array}{c}\text { Within-group } \\
P \text { values }\end{array}$ \\
\hline Supine systolic BP, mm Hg & $2.55(1.06)$ & $0.45,4.65$ & 0.018 & $0.52(1.02)$ & $-1.49,2.54$ & 0.607 \\
Supine Diastolic BP, mm Hg & $2.25(0.71)$ & $0.85,3.66$ & 0.002 & $-0.68(0.72)$ & $-2.12,0.75$ & 0.349 \\
Supine pulse rate, bpm & $7.33(0.88)$ & $5.60,9.06$ & $<0.001$ & $-1.15(0.97)$ & $-3.07,0.77$ & 0.238 \\
Weight, kg & $-0.83(0.49)$ & $-1.79,0.13$ & 0.091 & $-0.19(0.46)$ & $-1.11,0.73$ & 0.679 \\
\hline
\end{tabular}

${ }^{\mathrm{a}}$ Baseline for the extension phase was derived as the Week 10 value from the acute phase of the study. LS mean values were obtained from a mixed-effects repeated measures analysis. Abbreviations: BP, blood pressure; bpm, beats per minute; CI, 95\% confidence interval; EDX, edivoxetine; LS, least squares; n, number of patients at endpoint with the measure; PBO, placebo.

Table 5. Potentially clinically significant changes in vital signs and weight that occurred after the extension phase baseline ${ }^{\mathrm{a}}$.

\begin{tabular}{cccccc}
\hline & \multicolumn{2}{c}{ PBO/EDX } & \multicolumn{2}{c}{ EDX/EDX } \\
\hline Vital Sign & $\mathrm{N}$ & $\mathrm{n}(\%)$ & $\mathrm{N}$ & $\mathrm{n}(\%)$ \\
\hline Supine Systolic BP & 207 & $3(1.4)$ & 188 & $2(1.1)$ \\
Supine Diastolic BP & 207 & $5(2.4)$ & 188 & $1(0.5)$ \\
$\quad$ Supine Pulse & 207 & $1(0.5)$ & 188 & $1(0.5)$ \\
Weight, kg & & & & \\
Decrease $\geq 7 \%$ & 206 & $30(14.6)$ & 188 & $20(10.6)$ \\
Increase $\geq 7 \%$ & 206 & $14(6.8)$ & 188 & $16(8.5)$ \\
\hline
\end{tabular}

${ }^{a}$ Baseline for the extension phase was derived as the Week 10 value from the acute phase of the study. Parameters for potentially clinically significant: supine systolic BP: $\geq 180 \mathrm{~mm} \mathrm{Hg}$ and $\geq 20 \mathrm{~mm} \mathrm{Hg}$ increase from baseline; supine diastolic BP: $\geq 105 \mathrm{~mm} \mathrm{Hg}$ and increase from baseline $\geq 15 \mathrm{~mm} \mathrm{Hg}$; supine pulse: $>120$ beats per minute (bpm) and increase $\geq 15$ bpm from baseline. Abbreviations: BP, blood pressure; EDX, edivoxetine; N, number of patients with data; n, number of patients with categorical change; PBO, placebo.

Table 6. LS Mean Changes from Extension Phase Baseline ${ }^{\mathrm{a}}$ in Illness Severity.

\begin{tabular}{|c|c|c|c|c|c|c|}
\hline \multirow[b]{2}{*}{ Measure } & \multicolumn{2}{|c|}{ PBO/EDX } & \multicolumn{4}{|c|}{ EDX/EDX } \\
\hline & $\begin{array}{l}\text { LS Mean Change, } \\
\text { (SE) }\end{array}$ & CI & $\begin{array}{l}\text { Within-Group } \\
P \text { values }\end{array}$ & $\begin{array}{l}\text { LS Mean Change, } \\
\text { (SE) }\end{array}$ & CI & $\begin{array}{c}\text { Within-Group } \\
P \text { values }\end{array}$ \\
\hline MADRS Total & $-9.43(0.7)$ & $-10.8,-8.1$ & $<0.001$ & $-7.4(0.7)$ & $-8.8,-6.1$ & $<0.001$ \\
\hline CGI-Severity & $-1.24(0.1)$ & $-1.4,-1.1$ & $<0.001$ & $-1.03(0.1)$ & $-1.2,-0.9$ & $<0.001$ \\
\hline FAs-D PRO Average & $-0.65(0.1)$ & $-0.8,-0.5$ & $<0.001$ & $-0.5(0.8)$ & $-0.7,-0.4$ & $<0.001$ \\
\hline FAs-D Experience & $-0.7(0.1)$ & $-0.8,-0.6$ & $<0.001$ & $-0.5(0.1)$ & $-0.7,-0.3$ & $<0.001$ \\
\hline FAs-D Impact & $-0.58(0.1)$ & $-0.7,-0.4$ & $<0.001$ & $-0.51(-0.1)$ & $-0.7,-0.4$ & $<0.001$ \\
\hline SDS, Global & $-4.6(0.6)$ & $-5.7,-3.4$ & $<0.001$ & $-3.1(0.7)$ & $-4.4,-1.8$ & $<0.001$ \\
\hline
\end{tabular}

${ }^{\mathrm{a}}$ Baseline in the extension phase was derived as the Week 10 value from the acute phase of the study. LS mean values were obtained from a repeated measures analysis. Abbreviations: CGI-S, Clinical Global Impression of Severity; CI, 95\% confidence interval; EDX, edivoxetine; FAs-D PRO, Fatigue Associated with Depression Patient Reported Outcome; LS, least squares; MADRS, Montgomery-Åsberg Depression Rating Scale; PBO, placebo; SD, standard deviation; SDS, Sheehan Disability Scale.

quency than what was observed in the EDX/EDX group, but they were similar to the rates reported by edivoxetine-treated patients in the acute placebo-controlled phase of the study, as well as in another earlier study of edivoxetine in MDD [13]. The higher incidence of TEAEs in the PBO/EDX group during the extension phase may reflect the lack of prior exposure to edivoxetine; whereas, the lower incidence of TEAEs in the EDX/EDX group suggests that most TEAEs occur early in treatment and become less frequent over time.

The most common TEAEs reported during long-term treatment with edivoxetine were consistent with those reported in the acute phase of this study [5] and an earlier edivoxetine study [13], as well as other long-term MDD studies of antidepressants with norepinephrine re-uptake inhibition [14]-[22]. The frequency of SAE occurrence was low $(<3 \%)$, and there were no deaths during this extension phase. The mean increases in supine systolic and diastolic BP and pulse observed in the PBO/EDX group were consistent with the changes seen during the 10-week acute phase of this study in patients randomized to edivoxetine.The overall small $(<1 \mathrm{~mm}$ $\mathrm{Hg}$ ) mean changes in systolic and diastolic BP and small decrease in pulse rates observed in the EDX/EDX 
group suggest that the increase in these measures that occurred after initiating treatment with edivoxetine in the acute phase of the study had reached a plateau. The rate of PCS changes in systolic and diastolic blood pressure and pulse were numerically higher in the PBO/EDX group than in the EDX/EDX group. Overall, 4 of these patients completed the study; 5 patients discontinued due to adverse events: high blood pressure $(n=2)$, increased heart rate $(\mathrm{n}=1)$, palpitations $(\mathrm{n}=1)$, and insomnia $(\mathrm{n}=1)$.

Changes in weight, especially weight gain, are of concern to patients on antidepressant treatment [23]. Longterm treatment with edivoxetine was associated more frequently with weight loss than weight gain. Overall, the mean change in weight from the extension phase baseline was less than $1 \mathrm{~kg}$. For those patients who experienced a substantial $(\geq 7 \%)$ change in weight, approximately $13 \%$ lost weight and approximately $8 \%$ gained weight. These changes in weight were similar to what has been reported during long-term treatment with other antidepressants with norepinephrine reuptake inhibition, such as duloxetine [16] [17], venlafaxine [15], and levomilnacipran [24]

Erectile dysfunction in male patients is also another concern associated with antidepressant treatment [25]. Long-term exposure to edivoxetine in this study was associated with erectile dysfunction in $6.4 \%$ of male patients. This adverse event was the reason for study discontinuation in one patient, and a request for a reduction in edivoxetine dose for another patient. The occurrence of erectile dysfunction as an adverse event has been reported with similar frequency during long-term treatment with other antidepressants with norepinephrine reuptake inhibition [16] [22].

Although the outcome of the 10-week acute treatment phase of this study was a significantly greater reduction in depressive symptoms in edivoxetine- versus placebo-treated patients [5], the mean MADRS total was nearly 16 for the edivoxetine group that entered the extension phase, which suggests that some of these patients had residual symptoms. By the end of an additional 52 weeks of treatment, the mean MADRS total score in these patients had been reduced further by 7 points and $60.5 \%$ had remitted.

Some limitations to this study need to be taken into consideration when interpreting the results. Patients with an unstable medical illness, or some comorbid psychiatric and medical conditions were excluded. Given the high incidence of psychiatric conditions comorbid with MDD, the patient population in this report may not be fully representative of patients typically seen in clinical practice. This open-label extension phase did not include any comparator including placebo, so interpretation of the efficacy data cannot be attributed to specific causative factors.

\section{Conclusion}

In conclusion, the safety and tolerability findings during this long-term evaluation of edivoxetine were consistent with the safety profile reported in the acute phase of this study. In addition, these findings were comparable to reports on other antidepressants with norepinephrine reuptake inhibition.

\section{References}

[1] Hirschfeld, R.M. (2001) Clinical Importance of Long-Term Antidepressant Treatment. British Journal of Psychiatry, 42, S4-S8. http://dx.doi.org/10.1192/bjp.179.42.s4

[2] Greden, J.F. (2001) The Burden of Recurrent Depression: Causes, Consequences, and Future Prospects. Journal of Clinical Psychiatry, 62(Suppl 22), 5-9.

[3] Keller, M.B. (2002) Rationale and Options for the Long-Term Treatment of Depression. Human Psychopharmacology, 17, S43-S46. http://dx.doi.org/10.1002/hup.400

[4] Stahl, S.M., Zhang, L., Damatarca, C. and Grady, M. (2003) Brain Circuits Determine Destiny in Depression: A Novel Approach to the Psychopharmacology of Wakefulness, Fatigue, and Executive Dysfunction in Major Depressive Disorder. Journal of Clinical Psychiatry, 64(Suppl 14), 6-17.

[5] Pangallo, B., Dellva, M.A., D’Souza, D.N., Essink, B., Russell, J. and Goldberger, C. (2011) A Randomized, DoubleBlind Study Comparing LY2216684 and Placebo in the Treatment of Major Depressive Disorder. Journal of Psychiatric Research, 45, 748-755. http://dx.doi.org/10.1016/j.jpsychires.2011.03.014

[6] Sheehan, D.V., Lecrubier, Y., Sheehan, K.H., Amorim, P., Janavs, J., Weiller, E., Hergueta, T., Baker, R. and Dunbar, G.C. (1998) The Mini-International Neuropsychiatric Interview (M.I.N.I.): The Development and Validation of a Structured Diagnostic Psychiatric Interview for DSM-IV and ICD-10. Journal of Clinical Psychiatry, 59(Suppl 20), 22-33.

[7] Williams, J.B., Kobak, K.A., Bech, P., Engelhardt, H., Evans, K., Lipsitz, J., Olin, J., Pearson, J. and Kalali, A. (2008) 
The GRID-HAMD: Standardization of the Hamilton Depression Rating Scale. International Clinical Psychopharmacology, 23, 120-129. http://dx.doi.org/10.1097/YIC.0b013e3282f948f5

[8] Guy, W. (1976) Clinical Global Impressions, ECDEU Assessment Manual for Psychopharmacology, revised (DHEW Publ. No. ADM 76-338). National Institute of Mental Health, Rockville, 218-222.

[9] Posner, K., Brown, G.K., Stanley, B., Brent, D.A., Yershova, K.V., Oquendo, M.A., Currier, G.W., Melvin, G.A., Greenhill, L., Shen, S. and Mann, J.J. (2011) The Columbia-Suicide Severity Rating Scale: Initial Validity and Internal Consistency Findings from Three Multisite Studies with Adolescents and Adults. American Journal of Psychiatry, 168, 1266-1277. http://dx.doi.org/10.1176/appi.ajp.2011.10111704

[10] Montgomery, S.A. and Åsberg, M. (1979) A New Depression Scale Designed to be Sensitive to Change. British Journal of Psychiatry, 134, 382-389. http://dx.doi.org/10.1192/bjp.134.4.382

[11] Matza, L.S., Phillips, G.A., Revicki, D.A., Murray, L. and Malley, K.G. (2011) Development and Validation of a Patient-Report Measure of Fatigue Associated with Depression. Journalof Affective Disorders, 134, 294-303. http://dx.doi.org/10.1016/j.jad.2011.06.028

[12] Sheehan, D.V. (1983) The Anxiety Disease. Scribner, New York.

[13] Dubé, S., Dellva, M.A., Jones, M., Kielbasa, W., Padich, R., Saha, A. and Rao, P. (2010) A Study of the Effects of LY2216684, a Selective Norepinephrine Reuptake Inhibitor, in the Treatment of Major Depression. Journal of Psychiatric Research, 44, 356-363. http://dx.doi.org/10.1016/j.jpsychires.2009.09.013

[14] Versiani, M., Mehilane, L., Gaszner, P. and Arnaud-Castiglioni, R. (1999) Reboxetine, a Unique Selective NRI, Prevents Relapse and Recurrence in Long-Term Treatment of Major Depressive Disorder. Journal of Clinical Psychiatry, 60, 400-406. http://dx.doi.org/10.4088/JCP.v60n0610

[15] Schweitzer, I., Burrows, G., Tuckwell, V., Polonowita, A., Flynn, P., George, T., Theodoros, M. and Mitchell, P. (2001) Sustained Response to Open-Label Venlafaxine in Drug-Resistant Major Depression. Journal of Clinical Psychopharmacology, 21, 185-189. http://dx.doi.org/10.1097/00004714-200104000-00010

[16] Raskin, J., Goldstein, D.J., Mallinckrodt, C.H. and Ferguson, M.B. (2003) Duloxetine in the Long-Term Treatment of Major Depressive Disorder. Journal of Clinical Psychiatry, 64, 1237-1244. http://dx.doi.org/10.4088/JCP.v64n1015

[17] Wohlreich, M.M., Mallinckrodt, C.H., Watkin, J.G. and Hay, D.P. (2004) Duloxetine for the Long-Term Treatment of Major Depressive Disorder in Patients Aged 65 and Older: An Open-Label Study. BMC Geriatrics, 7, 4-11.

[18] Wade, A., Despiegel, N. and Heldbo Reines, E. (2006) Escitalopram in the Long-Term Treatment of Major Depressive Disorder. Annals of Clinical Psychiatry, 18, 83-89. http://dx.doi.org/10.1080/10401230600614447

[19] Chokka, P. and Legault, M. (2008) Escitalopram in the Treatment of Major Depressive Disorder in Primary-Care Settings: An Open-Label Trial. Depression and Anxiety, 25, E173-E181. http://dx.doi.org/10.1002/da.20458

[20] Dunner, D.L., Wilson, M., Fava, M., Kornstein, S., Munoz, R., O’Reardon, J., Trivedi, M. and Wohlreich, M. (2008) Long-Term Tolerability and Effectiveness of Duloxetine in the Treatment of Major Depressive Disorder. Depression and Anxiety, 25, E1-E8. http://dx.doi.org/10.1002/da.20339

[21] Tourian, K.A., Pitrosky, B., Padmanabhan, S.K. and Rosas, G.R. (2011) A 10-Month, Open-Label Evaluation of Desvenlafaxine in Outpatients with Major Depressive Disorder. Primary Care Companionto CNS Disorders, 13, ii: PCC.10m00977. http://dx.doi.org/10.4088/pcc.10m00977

[22] Mago, R., Forero, G., Greenberg, W.M., Gommoll, C. and Chen, C. (2013) Safety and Tolerability of Levomilnacipran ER in Major Depressive Disorder: Results from an Open-Label, 48-Week Extension Study. Clinical Drug Investigations, 33, 761-771. http://dx.doi.org/10.1007/s40261-013-0126-5

[23] Kelly, K., Posternak, M. and Alpert, J.E. (2008) Toward Achieving Optimal Response: Understanding and Managing Antidepressant Side Effects. Dialogues in Clinical Neuroscience, 10, 409-418.

[24] Citrome, L. (2013) Levomilnacipran for Major Depressive Disorder: A Systematic Review of the Efficacy and Safety Profile for This Newly Approved Antidepressant-What Is the Number Needed to Treat, Number Needed to Harm and Likelihood to be Helped or Harmed? International Journal of Clinical Practice, 67, 1089-10104. http://dx.doi.org/10.1111/ijcp.12298

[25] Serretti, A. and Chiesa, A. (2009) Treatment-Emergent Sexual Dysfunction Related to Antidepressants: A MetaAnalysis. Journal of Clinical Psychopharmacology, 29, 259-266. http://dx.doi.org/10.1097/JCP.0b013e3181a5233f 